\title{
Nonadiabatic dynamics in the semiclassical Liouville representation: Locality, transformation theory, and the energy budget
}

\author{
Craig C. Martens
}

Department of Chemistry, University of California, Irvine, California 92697-2025

\begin{abstract}
In this paper, we revisit the semiclassical Liouville approach to describing molecular dynamics with electronic transitions using classical trajectories. Key features of the formalism are highlighted. The locality in phase space and presence of nonclassical terms in the generalized Liouville equations are emphasized and discussed in light of trajectory surface hopping methodology. The representation dependence of the coupled semiclassical Liouville equations in the diabatic and adiabatic bases are discussed and new results for the transformation theory of the Wigner functions representing the corresponding density matrix elements given. We show that the diagonal energies of the state populations are not conserved during electronic transitions, as energy is stored in the electronic coherence. We discuss the implications of this observation for the validity of imposing strict energy conservation in trajectory based methods for simulating nonadiabatic processes.
\end{abstract}

\section{Introduction}

Physical phenomena on the molecular scale are described by quantum mechanics. Solving the time-dependent Schrödinger equation numerically has become a feasible approach for treating molecular dynamics of significant complexity, especially in cases where approximate methods and their systematic corrections can be applied efficiently $[1,2]$. This approach inevitably runs into problems, however, due to its unfavorable scaling with

Email address: cmartens@uci.edu (Craig C. Martens) 
system size. Alternative methods incorporating additional approximations must be employed to meet this challenge.

It sometimes works to simply ignore quantum effects completely. Classical molecular dynamics (MD) is routinely employed to treat many-atom systems $[3,4]$. In a classical MD simulation, an ensemble of trajectories is employed to represent a quantum mechanical wave packet, thermal distribution, or other initial equilibrium or nonequilibrium state, and the dynamics of the state is modeled by propagating the individual trajectories of the ensemble in phase space under Hamiltonian dynamics [5]. Classical trajectories can often do a remarkably good job of modeling the statistics and dynamics of molecular systems and reproducing the results of detailed experiments (see, e.g. [6]).

A purely classical treatment fails when the Born-Oppenheimer approximation breaks down and the classical nuclear dynamics are accompanied by intrinsically quantum mechanical nonadiabatic electronic transitions. A great deal of interest and activity has been focused on developing semiclassical or mixed quantum-classical methods for modeling molecular dynamics with electronic transitions. These include trajectory surface hopping $[7,8,9,10,11,12,13]$, full multiple spawning [14, 15], semiclassical initial value representation [16, 17], quantum hydrodynamics [18], mapping Hamiltonian [19], and linearized approaches [20, 21], to cite just a few. As part of this effort, we introduced an alternative approach based on a semiclassical limit of the quantum Liouville equation in the Wigner representation [22] which has been developed by us and others [23, 24, 25, 26, 27, 28, 29, 30]. In general, mixed quantum-classical methods hold great promise, as they allow the essential quantum features of complex systems to be incorporated within the computationally less demanding and intuitively appealing framework of classical mechanics.

In this paper we revisit the semiclassical Liouville formalism [23, 24, 25, $26,27,28,29,30$ ] from the perspective of ongoing developments in the general area of treating molecular dynamics with nonadiabatic electronic transitions. We consider the semiclassical limit of the multistate quantum Liouville equation in both the diabatic and adiabatic representations with a focus on the relation between the formal theory describing the evolution of the statesphase space generalized distribution functions - and methods to implement the theory with surface hopping trajectory ensembles. In particular, we discuss the locality of the equations of motion in phase space, nonclassical features of the dynamics, the transformation theory in the semiclassical Li- 
ouville representation, and the roles of energy conservation and electronic coherence in the formalism. Recent work has been directed at forging a connection between the semiclassical Liouville approach and Tully's fewest switches surface hopping (FSSH) [27, 11], and the goal of this paper is to contribute to that ongoing effort to undertand the fundamental challenges of representing quantum processes in a classical trajectory framework.

\section{Formalism}

We briefly review the semiclassical Liouville method for describing nuclear dynamics on coupled electronic states [22, 23, 24, 25, 26]. The starting point is the quantum mechanical Liouville equation for the density operator, $\hat{\rho}(t)$, describing dynamics on a single potential surface $U(q)$. For simplicity, we consider a single classical degree of freedom; the generalization to higher dimensions is straightforward. The quantum Liouville equation is

$$
i \hbar \frac{\partial \hat{\rho}}{\partial t}=[\hat{H}, \hat{\rho}]
$$

where $\hat{H}=\frac{\hat{p}^{2}}{2 m}+U(q)$ is the Hamiltonian of the system. The classical limit of Eq. (1) becomes the classical Liouville equation of non-equilibrium statistical mechanics [31]:

$$
\frac{\partial \rho}{\partial t}=\{H, \rho\}
$$

where $\rho=\rho(q, p, t)$ and $H=H(q, p)$ are now functions of the classical phase space variables $(q, p)$, and we assume that the Hamiltonian does not depend explicitly on time. The Poisson bracket on the right side of the equation is given by

$$
\{H, \rho\}=\frac{\partial H}{\partial q} \frac{\partial \rho}{\partial p}-\frac{\partial \rho}{\partial q} \frac{\partial H}{\partial p} .
$$

This result can be derived through the Wigner-Moyal expansion of the quantum mechanical Liouville equation $[32,33]$. To the lowest order in $\hbar$, the quantum commutator is replaced by the corresponding classical Poisson bracket:

$$
[\hat{H}, \hat{\rho}] \rightarrow i \hbar\{H, \rho\}+\mathcal{O}\left(\hbar^{3}\right) .
$$

The density operator is expressed in this representation as the Wigner function, given by $[32,33]$

$$
\rho(q, p)=\frac{1}{2 \pi \hbar} \int_{-\infty}^{\infty}\left\langle q-\frac{1}{2} y|\hat{\rho}| q+\frac{1}{2} y\right\rangle e^{i p y / \hbar} d y .
$$


This classical limit can be extended to the case where $\hat{H}$ is an $N \times N$ matrix of operators, representing an $N$ state quantum subsystem for which the classical limit is not taken $[22,23,24,25,26]$. For simplicity, our development will treat the case $N=2$. The Hamiltonian and density operator for the system can then be represented in a given quantum basis as:

$$
\hat{H}=\left(\begin{array}{ll}
\hat{H}_{11} & \hat{H}_{12} \\
\hat{H}_{21} & \hat{H}_{22}
\end{array}\right)
$$

and

$$
\hat{\rho}=\left(\begin{array}{ll}
\hat{\rho}_{11} & \hat{\rho}_{12} \\
\hat{\rho}_{21} & \hat{\rho}_{22}
\end{array}\right),
$$

respectively. The result is three coupled equations for the operator matrix elements,

$$
\begin{gathered}
i \hbar \frac{\partial \hat{\rho}_{11}}{\partial t}=\left[\hat{H}_{11}, \hat{\rho}_{11}\right]+\hat{H}_{12} \hat{\rho}_{21}-\hat{\rho}_{12} \hat{H}_{21} \\
i \hbar \frac{\partial \hat{\rho}_{22}}{\partial t}=\left[\hat{H}_{22}, \hat{\rho}_{22}\right]+\hat{H}_{21} \hat{\rho}_{12}-\hat{\rho}_{21} \hat{H}_{12} \\
i \hbar \frac{\partial \hat{\rho}_{12}}{\partial t}=\hat{H}_{11} \hat{\rho}_{12}+\hat{H}_{12} \hat{\rho}_{22}-\hat{\rho}_{11} \hat{H}_{12}-\hat{\rho}_{12} \hat{H}_{22},
\end{gathered}
$$

where $\hat{\rho}_{21}=\hat{\rho}_{12}^{\dagger}$.

\section{Diabatic Representation}

We first consider the case of individually Hermitian off-diagonal Hamiltonians, so $\hat{H}_{12}=\hat{H}_{21}=\hat{V}$. This is appropriate for a diabatic electronic basis. In the diabatic representation, the kinetic energy operator is diagonal, while the off-diagonal elements of the Hamiltonian are the function $V(q)$. The exact quantum equations of motion become

$$
\begin{gathered}
i \hbar \frac{\partial \hat{\rho}_{11}}{\partial t}=\left[\hat{H}_{11}, \hat{\rho}_{11}\right]+\hat{V} \hat{\rho}_{21}-\hat{\rho}_{12} \hat{V} \\
i \hbar \frac{\partial \hat{\rho}_{22}}{\partial t}=\left[\hat{H}_{22}, \hat{\rho}_{22}\right]+\hat{V} \hat{\rho}_{12}-\hat{\rho}_{21} \hat{V} \\
i \hbar \frac{\partial \hat{\rho}_{12}}{\partial t}=\hat{H}_{11} \hat{\rho}_{12}+\hat{V} \hat{\rho}_{22}-\hat{\rho}_{11} \hat{V}-\hat{\rho}_{12} \hat{H}_{22} .
\end{gathered}
$$

We now take the semiclassical limit of Eqs. (11)-(13) using the Wigner-Moyal representation of the product of operators in terms of the corresponding 
phase space functions obtained by Wigner transformation. We truncate the $\hbar$ expansion at first order, using the result [33]

$$
\hat{A} \hat{B}=A B+\frac{i \hbar}{2}\{A, B\}+\mathcal{O}\left(\hbar^{2}\right)
$$

where $A(q, p)$ and $B(q, p)$ are the phase space functions corresponding to the operators $\hat{A}$ and $\hat{B}$.

With the quantum mechanical operators replaced by the corresponding classical phase space functions in the semiclassical limit, the multistate semiclassical Liouville equations in the diabatic representation can be written as $[22,23,24,25,26]$ :

$$
\begin{gathered}
\frac{\partial \rho_{11}}{\partial t}=\left\{H_{11}, \rho_{11}\right\}+\left\{V, \operatorname{Re} \rho_{12}\right\}-\frac{2 V}{\hbar} \operatorname{Im} \rho_{12} \\
\frac{\partial \rho_{22}}{\partial t}=\left\{H_{22}, \rho_{22}\right\}+\left\{V, \operatorname{Re} \rho_{12}\right\}+\frac{2 V}{\hbar} \operatorname{Im} \rho_{12} \\
\frac{\partial \rho_{12}}{\partial t}=\left\{H_{o}, \rho_{12}\right\}-i \omega \rho_{12}+\frac{1}{2}\left\{V, \rho_{11}+\rho_{22}\right\}+\frac{i}{\hbar} V\left(\rho_{11}-\rho_{22}\right) .
\end{gathered}
$$

The Hamiltonian matrix elements $H_{i j}(q, p)$ and density matrix elements $\rho_{i j}(q, p, t)$ are now functions of the classical variables $(q, p)$. The function $H_{0}=\left(H_{11}+H_{22}\right) / 2$ is the average of the two uncoupled surface Hamiltonians, and the frequency term $\omega(q)$ is defined as the difference Hamiltonian divided by Planck's constant,

$$
\omega(q)=\frac{H_{11}(q, p)-H_{22}(q, p)}{\hbar} .
$$

The first term on the right side of Eqs. (15) and (16) corresponds to classical dynamics of the phase space probability densities, governed by the classical Hamiltonians $H_{11}$ and $H_{22}$. The remaining terms that involve the coupling matrix element $V$ and the coherence $\rho_{12}$ correspond to the nonclassical quantum coupling between the states, and include both sink and source terms that invoke transitions between phase space probability densities $\rho_{11}$ and $\rho_{22}$ and nonclassical modifications of the classical-limit Liouvillian dynamics on each state. In the equation of motion for the coherence, Eq. (17), the first two terms on the right side corresponds to a generalized classical dynamics, involving both propagation under the average Hamiltonian $H_{0}$ and accumulation of a nonclassical phase factor resulting from the imaginary frequency 
term. Unlike the diagonal probability densities, the coherence $\rho_{12}$ is thus a complex valued function. The densities $\rho_{11}$ and $\rho_{22}$ come in through the inhomogeneous terms that create and modify the evolving coherence.

The equations of motion for the semiclassical limit density matrix elements in the diabatic representation are composed of two types of terms. First, there are trace-preserving terms that do not change the total populations of the states, $\operatorname{Tr} \rho_{11}$ and $\operatorname{Tr} \rho_{22}$, or the total integrated coherence $\operatorname{Tr} \rho_{12}$, where $\operatorname{Tr} f \equiv \iint f(q, p) d q d p$. These terms consist of homogeneous contributions of the form $\{H, \rho\}$, which correspond to the classical dynamics of the Liouville equation, and the inhomogeneous terms, $\left\{V, \operatorname{Re} \rho_{12}\right\}$ for the populations and $\frac{1}{2}\left\{V, \rho_{11}+\rho_{22}\right\}$ for the coherence, which modify the classical evolution. In general, for functions $f$ and $g$, at least one of which vanishes at the boundaries of the region of integration in phase space, we have

$\operatorname{Tr}\{f, g\}=\iint\left(\frac{\partial f}{\partial q} \frac{\partial g}{\partial p}-\frac{\partial g}{\partial q} \frac{\partial f}{\partial p}\right) d q d p=-\iint f\left(\frac{\partial^{2} g}{\partial q \partial p}-\frac{\partial^{2} g}{\partial p \partial q}\right) d q d p=0$.

Here, we have integrated by parts and used the vanishing of $f$ at the boundary of phase space. Thus, the terms involving Poisson brackets in the equations of motion do not result in a change of the trace of the evolving phase space functions.

The second type of term consists of couplings that are not Poisson brackets, and that in general have a nonzero effect on the total populations and coherence. For the populations, these are the $\pm \frac{2 V}{\hbar} \operatorname{Im} \rho_{12}$ terms, while for the coherence this is the $\frac{i V}{\hbar}\left(\rho_{11}-\rho_{22}\right)$ term. These act as sink and source terms in phase space for the evolving probability densities, and in general capture the dynamics of electronic transitions in the diabatic representation.

An important property of the coupled semiclassical Liouville equations should be noted: these partial differential equations are local in phase space. The time rate of change of the generalized distribution function $\rho_{i j}(q, p, t)$ at the point $(q, p)$ depends on the full set of functions and their derivatives at that same point. This may seem an obvious feature of the equations, but the implications of this natural structure on trajectory-based approaches to solving them has not been emphasized. In particular, the analysis suggest that trajectories that undergo a stochastic "hop" between the states should mirror the locality of the underlying equations for the state. The standard surface hopping methods do not impose this locality, but rather introduce nonlocality through momentum rescaling to impose strict energy conserva- 
tion.

\section{Adiabatic Representation}

The semiclassical Liouville equations can also be formulated in the adiabatic basis $[24,29]$. In terms of the diabatic states $|1\rangle$ and $|2\rangle$ the quantum Hamiltonian operator in the position representation is

$$
\hat{H}=\hat{T} \mathbf{1}+\mathbf{U}(q)=\left(\begin{array}{lr}
\hat{T}+E_{1}(q) & V(q) \\
V(q) & \hat{T}+E_{2}(q)
\end{array}\right)
$$

where $\hat{T}=\hat{p}^{2} / 2 m=-\left(\hbar^{2} / 2 m\right) \partial^{2} / \partial q^{2}$ is the nuclear kinetic energy operator, $E_{j}(q)(j=1,2)$ are the diabatic potential curves, and $V(q)$ is the off-diagonal coupling between the diabatic electronic states. The adiabatic energies $E_{+}$ and $E_{-}$and eigenstates $|+\rangle$and $|-\rangle$are found by diagonalizing the potential energy matrix $\mathbf{U}(q)$ at each value of $q$. The results are

$$
\begin{gathered}
E_{ \pm}=\frac{E_{1}+E_{2}}{2} \pm \sqrt{\left(\frac{E_{1}-E_{2}}{2}\right)^{2}+V^{2}} \\
|+\rangle=|1\rangle \cos (\phi / 2)+|2\rangle \sin (\phi / 2) \\
|-\rangle=-|1\rangle \sin (\phi / 2)+|2\rangle \cos (\phi / 2),
\end{gathered}
$$

where the mixing angle $\phi(q)$ is given by

$$
\tan \phi(q)=\frac{2 V(q)}{E_{1}(q)-E_{2}(q)}
$$

In the adiabatic representation, the potential energy is diagonal. Offdiagonal terms in the Hamiltonian result from the nuclear kinetic energy operator $\hat{T}$. We can evaluate the matrix elements of $\hat{T}$ between the adiabatic states by expressing them in terms of the coordinate-independent diabatic states and computing the action of $\hat{T}$ explicitly. The results are

$$
\begin{gathered}
\hat{W}=\langle+|\hat{T}|-\rangle=\frac{i \hbar}{2 m} \phi^{\prime}(q) \hat{p}+\frac{\hbar^{2}}{4 m} \phi^{\prime \prime}(q) \\
\hat{W}^{\dagger}=\langle-|\hat{T}|+\rangle=-\frac{i \hbar}{2 m} \phi^{\prime}(q) \hat{p}-\frac{\hbar^{2}}{4 m} \phi^{\prime \prime}(q),
\end{gathered}
$$


where prime indicates derivative with respect to $q$. These are quantum mechanical operators that act on functions of the nuclear coordinate $q$. The second term proportional to $\hbar^{2}$ in each expression results from the second derivative of the adiabatic state with respect to $q$, and is needed to give a Hermitian Hamiltonian by compensating for the noncommutativity of $\hat{p}$ with $\phi^{\prime}(q)$. In the semiclassical limit, $\hat{p} \rightarrow p$, the classical canonical momentum, while the $\hbar^{2}$ terms are neglected. The off-diagonal nonadiabatic coupling then becomes a classical function of $q$ and $p$ : $W(q, p)=\frac{i \hbar}{2 m} \phi^{\prime}(q) p$. This function is purely imaginary.

To make connection with standard notation for nonadiabatic dynamics, we note the relation between our expressions and the so-called nonadiabatic coupling matrix element $d(q)$, defined as

$$
d(q) \equiv\left\langle+\left|\frac{\partial}{\partial q}\right|-\right\rangle .
$$

This can be easily evaluated for the nonadiabatic states in terms of the diabatic states and position-dependent angle $\phi(q)$, yielding the simple result

$$
d(q)=-\frac{\phi^{\prime}(q)}{2}
$$

In terms of this quantity, the off-diagonal element $\hat{W}=\langle+|\hat{H}|-\rangle$ can be written as

$$
\hat{W}=-\frac{i \hbar}{2}\left(d(q) \frac{\hat{p}}{m}+\frac{\hat{p}}{m} d(q)\right)
$$

with $\hat{W}^{\dagger}=\langle-|\hat{H}|+\rangle=-\hat{W}$, or in the semiclassical limit,

$$
W(q, p)=-i \hbar d(q) \frac{p}{m}
$$

with $W^{*}(q, p)=-W(q, p)$.

The density operator can also be expressed in the adiabatic representation:

$$
\hat{\rho}=\left(\begin{array}{cc}
\hat{\rho}_{++} & \hat{\rho}_{+-} \\
\hat{\rho}_{-+} & \hat{\rho}_{--}
\end{array}\right)
$$

The quantum Liouville equation is again

$$
i \hbar \frac{\partial \hat{\rho}}{\partial t}=[\hat{H}, \hat{\rho}]
$$


or written out term-by term in the adiabatic representation,

$$
\begin{gathered}
i \hbar \frac{\partial \hat{\rho}_{++}}{\partial t}=\left[\hat{H}_{++}, \hat{\rho}_{++}\right]+\hat{W} \hat{\rho}_{-+}-\hat{\rho}_{+-} \hat{W}^{\dagger} \\
i \hbar \frac{\partial \hat{\rho}_{--}}{\partial t}=\left[\hat{H}_{--}, \hat{\rho}_{--}\right]+\hat{W}^{\dagger} \hat{\rho}_{+-}-\hat{\rho}_{-+} \hat{W} \\
i \hbar \frac{\partial \hat{\rho}_{+-}}{\partial t}=\hat{H}_{++} \hat{\rho}_{+-}+\hat{W} \hat{\rho}_{--}-\hat{\rho}_{++} \hat{W}-\hat{\rho}_{+-} \hat{H}_{--} .
\end{gathered}
$$

We can now perform the Wigner transform to lowest order in $\hbar$ using $\hat{A} \hat{B}=$ $A B+\frac{i \hbar}{2}\{A, B\}+\mathcal{O}\left(\hbar^{2}\right)$ and $W(q, p)=-i \hbar d(q) p / m$. We obtain

$$
\begin{gathered}
\frac{\partial \rho_{++}}{\partial t}=\left\{H_{++}, \rho_{++}\right\}-2 d \frac{p}{m} \operatorname{Re} \rho_{+-}-\hbar\left\{d \frac{p}{m}, \operatorname{Im} \rho_{+-}\right\} \\
\frac{\partial \rho_{--}}{\partial t}=\left\{H_{--}, \rho_{--}\right\}+2 d \frac{p}{m} \operatorname{Re} \rho_{+-}-\hbar\left\{d \frac{p}{m}, \operatorname{Im} \rho_{+-}\right\} \\
\frac{\partial \rho_{+-}}{\partial t}=\left\{H_{o}, \rho_{+-}\right\}-i \omega \rho_{+-}+d \frac{p}{m}\left(\rho_{++}-\rho_{--}\right)-\frac{i \hbar}{2}\left\{d \frac{p}{m}, \rho_{++}+\rho_{--}\right\},
\end{gathered}
$$

where $H_{++}(q, p)=p^{2} / 2 m+E_{+}(q), H_{--}(q, p)=p^{2} / 2 m+E_{-}(q), H_{o}=$ $\frac{1}{2}\left(H_{++}+H_{--}\right), \omega(q)=\left(E_{+}(q)-E_{-}(q)\right) / \hbar$, and again $\{f, g\}$ is the Poisson bracket of $f(q, p)$ and $g(q, p)$.

In the above analysis, we express the quantum Hamiltonian in the adiabatic representation from the outset, leading to a diagonal potential energy but a non-diagonal kinetic energy in the basis of adiabatic electronic states. We then perform the semiclassical limit analysis in that representation. An alternative approach followed in the literature is to perform the semiclassical limit first and then represent the quantum-classical equations of motion for the Wigner function in the adiabatic basis. The basis tranformation and semiclassical limit do not commute, and the two alternatives yield somewhat different results. Kapral and coworkers follow the semiclassical limit then adiabatic representation path in their development [27], and recently discussed issues arising in systems with conical intersections exhibiting Berry's phase [34]. Ando and coworkers follow the adiabatic then semiclassical approach that we employ in the present context $[29,30]$. We favor this approach, as the approximations inherent in the semiclassical limit are introduced as the final, rather than an intermediate, step. 


\section{Transformation Theory in the Semiclassical Liouville Represen- tation}

We now investigate the transformation from diabatic to adiabatic electronic bases in the Wigner representation and derive new results for the transformation equations.

The adiabatic states $\psi_{+}(q)$, and $\psi_{-}(q)$ are given in terms of the diabatic states $\psi_{1}(q)$ and $\psi_{2}(q)$ as

$$
\begin{gathered}
\psi_{+}(q)=\cos \left(\frac{\phi(q)}{2}\right) \psi_{1}(q)+\sin \left(\frac{\phi(q)}{2}\right) \psi_{2}(q) \\
\psi_{-}(q)=-\sin \left(\frac{\phi(q)}{2}\right) \psi_{1}(q)+\cos \left(\frac{\phi(q)}{2}\right) \psi_{2}(q) .
\end{gathered}
$$

The position representation of the density matrix elements in the adiabatic representation can then be constructed in terms of the diabatic representation elements:

$$
\begin{aligned}
\rho_{++}\left(q, q^{\prime}\right) & =c(q) c\left(q^{\prime}\right) \rho_{11}\left(q, q^{\prime}\right)+c(q) s\left(q^{\prime}\right) \rho_{12}\left(q, q^{\prime}\right) \\
& +s(q) c\left(q^{\prime}\right) \rho_{21}\left(q, q^{\prime}\right)+s(q) s\left(q^{\prime}\right) \rho_{22}\left(q, q^{\prime}\right) \\
\rho_{+-}\left(q, q^{\prime}\right) & =-c(q) s\left(q^{\prime}\right) \rho_{11}\left(q, q^{\prime}\right)+c(q) c\left(q^{\prime}\right) \rho_{12}\left(q, q^{\prime}\right) \\
& -s(q) s\left(q^{\prime}\right) \rho_{21}\left(q, q^{\prime}\right)+s(q) c\left(q^{\prime}\right) \rho_{22}\left(q, q^{\prime}\right) \\
\rho_{-+}\left(q, q^{\prime}\right) & =-s(q) c\left(q^{\prime}\right) \rho_{11}\left(q, q^{\prime}\right)-s(q) s\left(q^{\prime}\right) \rho_{12}\left(q, q^{\prime}\right) \\
& +c(q) c\left(q^{\prime}\right) \rho_{21}\left(q, q^{\prime}\right)+c(q) s\left(q^{\prime}\right) \rho_{22}\left(q, q^{\prime}\right) \\
\rho_{--}\left(q, q^{\prime}\right) & =s(q) s\left(q^{\prime}\right) \rho_{11}\left(q, q^{\prime}\right)-s(q) c\left(q^{\prime}\right) \rho_{12}\left(q, q^{\prime}\right) \\
& -c(q) s\left(q^{\prime}\right) \rho_{21}\left(q, q^{\prime}\right)+c(q) c\left(q^{\prime}\right) \rho_{22}\left(q, q^{\prime}\right),
\end{aligned}
$$

where $c(q)=\cos \left(\frac{\phi(q)}{2}\right), s(q)=\sin \left(\frac{\phi(q)}{2}\right)$, and $\rho_{i j}\left(q, q^{\prime}\right)=\psi_{i}(q) \psi_{j}^{*}\left(q^{\prime}\right)$. We can now compute the Wigner transform of $\rho_{++}\left(q, q^{\prime}\right)$ explicitly:

$$
\rho_{++}^{W}(q, p)=\frac{1}{2 \pi \hbar} \int_{-\infty}^{\infty} \rho_{++}\left(q-\frac{1}{2} y, q+\frac{1}{2} y\right) e^{i p y / \hbar} d y .
$$


(In this Section, Wigner functions are indicated as $\rho^{W}$ to distinguish them from density matrix elements in the position representation.) The general term corresponds to the Fourier transform of the product of two functions of $q$ and $y$ over the variable $y$, yielding the convolution of the corresponding Wigner functions with respect to the momentum $p$ :

$$
\frac{1}{2 \pi \hbar} \int_{-\infty}^{\infty} \xi_{i j}(q, y) \rho_{i j}\left(q-\frac{1}{2} y, q+\frac{1}{2} y\right) e^{i p y / \hbar} d y=\int_{-\infty}^{\infty} \xi_{i j}^{W}\left(q, p^{\prime}\right) \rho_{i j}^{W}\left(q, p-p^{\prime}\right) d p^{\prime}
$$

where $\xi_{i j}(q, y)$ is the corresponding product of transformation elements evaluated at $q \pm \frac{y}{2}$.

We now evaluate the transformation explicitly for the case of $\rho_{++}$. The resulting expression in terms of the expansion in the diabatic density matrix has 4 terms. We consider each separately. The first term is

$$
\operatorname{term}_{1}=\frac{1}{2 \pi \hbar} \int_{-\infty}^{\infty} c\left(q-\frac{1}{2} y\right) \rho_{11}\left(q-\frac{1}{2} y, q+\frac{1}{2} y\right) c\left(q+\frac{1}{2} y\right) e^{i p y / \hbar} d y .
$$

The trigonometric factor can be collected as

$$
\begin{aligned}
c\left(q-\frac{1}{2} y\right) c\left(q+\frac{1}{2} y\right) & =\frac{1}{2} \cos \left(\frac{\phi\left(q-\frac{1}{2} y\right)+\phi\left(q+\frac{1}{2} y\right)}{2}\right) \\
& +\frac{1}{2} \cos \left(\frac{\phi\left(q-\frac{1}{2} y\right)-\phi\left(q+\frac{1}{2} y\right)}{2}\right) .
\end{aligned}
$$

To make progress we introduce further approximations by expanding the arguments of the trigonometric functions in powers of $y$ and keep only the leading terms. Thus,

$$
\begin{gathered}
\frac{\phi\left(q-\frac{1}{2} y\right)+\phi\left(q+\frac{1}{2} y\right)}{2}=\phi(q)+\mathcal{O}\left(y^{2}\right) \\
\frac{\phi\left(q-\frac{1}{2} y\right)-\phi\left(q+\frac{1}{2} y\right)}{2}=-\frac{1}{2} \phi^{\prime}(q) y+\mathcal{O}\left(y^{3}\right) \simeq d(q) y .
\end{gathered}
$$

Term 1 can then be written approximately as

$$
\operatorname{term}_{1} \simeq \frac{1}{2 \pi \hbar} \int_{-\infty}^{\infty} \frac{1}{2}[\cos \phi(q)+\cos (d(q) y)] \rho_{11}\left(q-\frac{1}{2} y, q+\frac{1}{2} y\right) e^{i p y / \hbar} d y
$$

and evaluated, with the result

$$
\operatorname{term}_{1} \simeq \frac{1}{2} \rho_{11}^{W}(q, p) \cos \phi(q)+\frac{1}{4} \rho_{11}^{W}(q, p+\hbar d(q))+\frac{1}{4} \rho_{11}^{W}(q, p-\hbar d(q)),
$$


where $\rho_{11}^{W}(q, p)$ is the Wigner function corresponding to the diabatic density matrix element $\rho_{11}\left(q, q^{\prime}\right)$. A similar analysis gives

$$
\begin{aligned}
\operatorname{term}_{2} & \simeq \frac{1}{2} \rho_{12}^{W}(q, p) \sin \phi(q)+\frac{i}{4} \rho_{12}^{W}(q, p+\hbar d(q))-\frac{i}{4} \rho_{12}^{W}(q, p-\hbar d(q)) \\
\operatorname{term}_{3} & \simeq \frac{1}{2} \rho_{21}^{W}(q, p) \sin \phi(q)-\frac{i}{4} \rho_{21}^{W}(q, p+\hbar d(q))+\frac{i}{4} \rho_{21}^{W}(q, p-\hbar d(q)) \\
\operatorname{term}_{4} & \simeq-\frac{1}{2} \rho_{22}^{W}(q, p) \cos \phi(q)+\frac{1}{4} \rho_{22}^{W}(q, p+\hbar d(q))+\frac{1}{4} \rho_{22}^{W}(q, p-\hbar d(q)) .
\end{aligned}
$$

We now summarize the results of the transformations for all of the adiabatic density matrix elements. Introducing the shorthand notation $f=f(q, p)$ and $f( \pm)=f(q, p \pm \hbar d(q))$, we have

$$
\begin{aligned}
\rho_{++}^{W} & =\frac{1}{4}\left[\rho_{11}^{W}(+)+\rho_{11}^{W}(-)+\rho_{22}^{W}(+)+\rho_{22}^{W}(-)\right]+\frac{1}{2}\left(\rho_{11}^{W}-\rho_{22}^{W}\right) \cos \phi \\
& +\operatorname{Re} \rho_{12}^{W} \sin \phi-\frac{1}{2}\left[\operatorname{Im} \rho_{12}^{W}(+)-\operatorname{Im} \rho_{12}^{W}(-)\right] \\
\rho_{+-}^{W} & =-\frac{1}{2}\left(\rho_{11}^{W}-\rho_{22}^{W}\right) \sin \phi+\operatorname{Re} \rho_{12}^{W} \cos \phi+\frac{i}{2}\left[\operatorname{Im} \rho_{12}^{W}(+)+\operatorname{Im} \rho_{12}^{W}(-)\right] \\
& -\frac{i}{4}\left[\rho_{11}^{W}(+)-\rho_{11}^{W}(-)+\rho_{22}^{W}(+)-\rho_{22}^{W}(-)\right] \\
\rho_{-+}^{W} & =-\frac{1}{2}\left(\rho_{11}^{W}-\rho_{22}^{W}\right) \sin \phi+\operatorname{Re} \rho_{12}^{W} \cos \phi-\frac{i}{2}\left[\operatorname{Im} \rho_{12}^{W}(+)+\operatorname{Im} \rho_{12}^{W}(-)\right] \\
+ & \frac{i}{4}\left[\rho_{11}^{W}(+)-\rho_{11}^{W}(-)+\rho_{22}^{W}(+)-\rho_{22}^{W}(-)\right] \\
\rho_{--}^{W} & =\frac{1}{4}\left[\rho_{11}^{W}(+)+\rho_{11}^{W}(-)+\rho_{22}^{W}(+)+\rho_{22}^{W}(-)\right]-\frac{1}{2}\left(\rho_{11}^{W}-\rho_{22}^{W}\right) \cos \phi \\
& -\operatorname{Re} \rho_{12}^{W} \sin \phi-\frac{1}{2}\left[\operatorname{Im} \rho_{12}^{W}(+)-\operatorname{Im} \rho_{12}^{W}(-)\right] .
\end{aligned}
$$

These results reveal that nonlocal momentum "jumps" appear in the transformation equations: the phase space density matrix elements in the adiabatic representation at point $(q, p)$ depend on the diabatic phase space matrix elements at $(q, p)$ and also at $(q, p \pm \hbar d(q))$. The nonadiabatic coupling matrix element $d(q)$ appears explicitly in the transformations. We emphasize 
that these jumps are not dynamical displacements of the momentum, which play a role in higher order quantum effects in the evolution of the Wigner function, but rather are kinematic effects resulting from the transformation between bases [32].

In the spirit of the semiclassical limit, we can continue to make approximations by expanding the $\hbar$-dependent momentum shifts in a series in $\hbar$ and keep only the first term. For instance, we have

$$
\rho_{11}^{W}(q, p+\hbar d(q)) \simeq \rho_{11}^{W}(q, p)+\hbar d(q) \frac{\partial \rho_{11}^{W}(q, p)}{\partial p}=\rho_{11}^{W}-\frac{\hbar}{2}\left\{\phi, \rho_{11}^{W}\right\} .
$$

Putting the pieces together, we have the semiclassical limit transformation law for $\rho_{++}^{W}$ :

$$
\rho_{++}^{W}=\frac{1}{2}\left(\rho_{11}^{W}+\rho_{22}^{W}\right)+\frac{1}{2}\left(\rho_{11}^{W}-\rho_{22}^{W}\right) \cos \phi+\operatorname{Re} \rho_{12}^{W} \sin \phi+\frac{\hbar}{2}\left\{\phi, \operatorname{Im} \rho_{12}^{W}\right\}
$$

Using a similar approach, we find:

$$
\begin{aligned}
& \rho_{+-}^{W}=-\frac{1}{2}\left(\rho_{11}^{W}-\rho_{22}^{W}\right) \sin \phi+\operatorname{Re} \rho_{12}^{W} \cos \phi+i \operatorname{Im} \rho_{12}^{W}+\frac{i \hbar}{4}\left\{\phi, \rho_{11}^{W}+\rho_{22}^{W}\right\} \\
& \rho_{-+}^{W}=-\frac{1}{2}\left(\rho_{11}^{W}-\rho_{22}^{W}\right) \sin \phi+\operatorname{Re} \rho_{12}^{W} \cos \phi-i \operatorname{Im} \rho_{12}^{W}-\frac{i \hbar}{4}\left\{\phi, \rho_{11}^{W}+\rho_{22}^{W}\right\} \\
& \rho_{--}^{W}=\frac{1}{2}\left(\rho_{11}^{W}+\rho_{22}^{W}\right)-\frac{1}{2}\left(\rho_{11}^{W}-\rho_{22}^{W}\right) \cos \phi-\operatorname{Re} \rho_{12}^{W} \sin \phi+\frac{\hbar}{2}\left\{\phi, \operatorname{Im} \rho_{12}^{W}\right\}
\end{aligned}
$$

We note that these equations are closely related to the transformation equations for the diagonal density matrix elements in the adiabatic representation. For instance, Eq. (41) gives, for $q=q^{\prime}$ and defining $\rho_{++}=\rho_{++}(q, q)$, $\rho_{11}=\rho_{11}(q, q)$, etc.,

$$
\rho_{++}=\frac{1}{2}\left(\rho_{11}+\rho_{22}\right)+\frac{1}{2}\left(\rho_{11}-\rho_{22}\right) \cos \phi+\operatorname{Re} \rho_{12} \sin \phi .
$$

The other configuration space generalized density equations can be obtained by ignoring the $\hbar$ dependent terms above. The momentum jumps or limiting forms in terms of Poisson brackets in the Wigner transformed adiabatic transformation equations take into account the effect of the position dependence of the unitary transformation on the phase space functions. 
We can derive the reverse transformation from the adiabatic to diabatic representation. We start with the relations

$$
\begin{aligned}
\rho_{11}\left(q, q^{\prime}\right) & =c(q) c\left(q^{\prime}\right) \rho_{++}\left(q, q^{\prime}\right)-c(q) s\left(q^{\prime}\right) \rho_{+-}\left(q, q^{\prime}\right) \\
& -s(q) c\left(q^{\prime}\right) \rho_{-+}\left(q, q^{\prime}\right)+s(q) s\left(q^{\prime}\right) \rho_{--}\left(q, q^{\prime}\right) \\
\rho_{12}\left(q, q^{\prime}\right) & =c(q) s\left(q^{\prime}\right) \rho_{++}\left(q, q^{\prime}\right)+c(q) c\left(q^{\prime}\right) \rho_{+-}\left(q, q^{\prime}\right) \\
& -s(q) s\left(q^{\prime}\right) \rho_{-+}\left(q, q^{\prime}\right)-s(q) c\left(q^{\prime}\right) \rho_{--}\left(q, q^{\prime}\right) \\
\rho_{21}\left(q, q^{\prime}\right) & =s(q) c\left(q^{\prime}\right) \rho_{++}\left(q, q^{\prime}\right)-s(q) s\left(q^{\prime}\right) \rho_{+-}\left(q, q^{\prime}\right) \\
& +c(q) c\left(q^{\prime}\right) \rho_{-+}\left(q, q^{\prime}\right)-c(q) s\left(q^{\prime}\right) \rho_{--}\left(q, q^{\prime}\right) \\
\rho_{22}\left(q, q^{\prime}\right) & =s(q) s\left(q^{\prime}\right) \rho_{++}\left(q, q^{\prime}\right)+s(q) c\left(q^{\prime}\right) \rho_{+-}\left(q, q^{\prime}\right) \\
& +c(q) s\left(q^{\prime}\right) \rho_{-+}\left(q, q^{\prime}\right)+c(q) c\left(q^{\prime}\right) \rho_{--}\left(q, q^{\prime}\right) .
\end{aligned}
$$

These can be obtained from scratch or by noting that by letting $\phi \rightarrow-\phi$ the reverse transformations are obtained. Thus, we can write down the Wigner transform relations:

$$
\begin{aligned}
\rho_{11}^{W} & =\frac{1}{4}\left[\rho_{++}^{W}(+)+\rho_{++}^{W}(-)+\rho_{--}^{W}(+)+\rho_{--}^{W}(-)\right]+\frac{1}{2}\left(\rho_{++}^{W}-\rho_{--}^{W}\right) \cos \phi \\
& -\operatorname{Re} \rho_{+-}^{W} \sin \phi+\frac{1}{2}\left[\operatorname{Im} \rho_{+-}^{W}(+)-\operatorname{Im} \rho_{+-}^{W}(-)\right] \\
\rho_{12}^{W} & =\frac{1}{2}\left(\rho_{++}^{W}-\rho_{--}^{W}\right) \sin \phi+\operatorname{Re} \rho_{+-}^{W} \cos \phi+\frac{i}{2}\left[\operatorname{Im} \rho_{+-}^{W}(+)+\operatorname{Im} \rho_{+-}^{W}(-)\right] \\
& +\frac{i}{4}\left[\rho_{++}^{W}(+)-\rho_{++}^{W}(-)+\rho_{--}^{W}(+)-\rho_{--}^{W}(-)\right] \\
\rho_{21}^{W} & =\frac{1}{2}\left(\rho_{++}^{W}-\rho_{--}^{W}\right) \sin \phi+\operatorname{Re} \rho_{+-}^{W} \cos \phi-\frac{i}{2}\left[\operatorname{Im} \rho_{+-}^{W}(+)+\operatorname{Im} \rho_{+-}^{W}(-)\right] \\
& -\frac{i}{4}\left[\rho_{++}^{W}(+)-\rho_{++}^{W}(-)+\rho_{--}^{W}(+)-\rho_{--}^{W}(-)\right] \\
\rho_{22}^{W} & =\frac{1}{4}\left[\rho_{++}^{W}(+)+\rho_{++}^{W}(-)+\rho_{--}^{W}(+)+\rho_{--}^{W}(-)\right]-\frac{1}{2}\left(\rho_{++}^{W}-\rho_{--}^{W}\right) \cos \phi \\
& +\operatorname{Re} \rho_{+-}^{W} \sin \phi+\frac{1}{2}\left[\operatorname{Im} \rho_{+-}^{W}(+)-\operatorname{Im} \rho_{+-}^{W}(-)\right],
\end{aligned}
$$


and, in the small $\hbar$ limit,

$$
\begin{aligned}
& \rho_{11}^{W}=\frac{1}{2}\left(\rho_{++}^{W}+\rho_{--}^{W}\right)+\frac{1}{2}\left(\rho_{++}^{W}-\rho_{--}^{W}\right) \cos \phi-\operatorname{Re} \rho_{+-}^{W} \sin \phi-\frac{\hbar}{2}\left\{\phi, \operatorname{Im} \rho_{+-}^{W}\right\} \\
& \rho_{12}^{W}=\frac{1}{2}\left(\rho_{++}^{W}-\rho_{--}^{W}\right) \sin \phi+\operatorname{Re} \rho_{+-}^{W} \cos \phi+i \operatorname{Im} \rho_{+-}^{W}-\frac{i \hbar}{4}\left\{\phi, \rho_{++}^{W}+\rho_{--}^{W}\right\}(75) \\
& \rho_{21}^{W}=\frac{1}{2}\left(\rho_{++}^{W}-\rho_{--}^{W}\right) \sin \phi+\operatorname{Re} \rho_{+-}^{W} \cos \phi-i \operatorname{Im} \rho_{+-}^{W}+\frac{i \hbar}{4}\left\{\phi, \rho_{++}^{W}+\rho_{--}^{W}\right\} \quad(76) \\
& \rho_{22}^{W}=\frac{1}{2}\left(\rho_{++}^{W}+\rho_{--}^{W}\right)-\frac{1}{2}\left(\rho_{++}^{W}-\rho_{--}^{W}\right) \cos \phi+\operatorname{Re} \rho_{+-}^{W} \sin \phi-\frac{\hbar}{2}\left\{\phi, \operatorname{Im} \rho_{+-}^{W}\right\} .
\end{aligned}
$$

The transformation equations between representations introduce a nonlocality in the underlying phase space. For concreteness, consider the transformation from the diabatic representation to the adiabatic representation given in Eqs. (56)-(59). Each of the Wigner functions representing the density matrix elements in the adiabatic representation are given at phase space point $(q, p)$ by the combination of the corresponding diabatic Wigner functions sampled at 3 distinct phase space points: a local term $(q, p)$ and nonlocal contributions from $(q, p+\hbar d(q))$ and $(q, p-\hbar d(q))$. These "momentum jumps" are not the result of the dynamics of nonadiabatic transitions but rather come about through the kinematics of the transformation between representations. In particular, the trigonometric functions of the mixing angle $\phi(q)$ that multiply the density matrix elements $\rho_{i j}\left(q, q^{\prime}\right)$ in the position representation generate sum and difference "frequency" terms via a heterodyne-like phenomena in the Fourier transform in the definition of the Wigner function. Similar momentum displacements appear in the inverse transformation from the adiabatic to the diabatic representation, Eqs. (70)-(73).

These transformation expressions are rigorously invertible. By applying the transformations sequentially, it can be verified that the nonlocal terms cancel, leading to the original representation. In the small $\hbar$ limit, the jumps lead to Poisson bracket terms involving the mixing angle $\phi$. In this limit, it can be shown that the sequential transformations lead to residual terms of order $\hbar^{2}$, consistent with neglecting such terms in approximations made.

\section{The Energy Budget in the Nonadiabatic Transitions}

We now analyze the total energy of the system and its partitioning between the coupled states during molecular dynamics with nonadiabatic elec- 
tronic transitions. We consider the system in both the diabatic and adiabatic representations.

The Hamiltonian and density matrix in the semiclassical limit and the diabatic representation are given by

$$
H=\left(\begin{array}{ll}
H_{11} & V \\
V & H_{22}
\end{array}\right)
$$

and

$$
\rho=\left(\begin{array}{ll}
\rho_{11} & \rho_{12} \\
\rho_{21} & \rho_{22}
\end{array}\right)
$$

respectively. The total conserved energy of the system is given by the trace of the product of $H$ and $\rho$. In the semiclassical limit, this is given by the integral over phase space of the sum of the diagonal elements of $H \rho$. The matrix is

$$
H \rho=\left(\begin{array}{cc}
H_{11} \rho_{11}+V \rho_{21} & H_{11} \rho_{12}+V \rho_{22} \\
V \rho_{11}+H_{22} \rho_{21} & V \rho_{12}+H_{22} \rho_{22}
\end{array}\right)
$$

and so Tr $H \rho$ becomes

$$
E=\operatorname{Tr} H \rho=\iint\left(H_{11} \rho_{11}+H_{22} \rho_{22}+2 V \operatorname{Re} \rho_{12}\right) d q d p .
$$

The energy is a sum of three terms: $E=E_{1}+E_{2}+E_{c o h}$. The first two terms are the average energies of the system on state 1 and state 2 , respectively:

$$
\begin{aligned}
& E_{1}=\iint H_{11} \rho_{11} d q d p \\
& E_{2}=\iint H_{22} \rho_{22} d q d p
\end{aligned}
$$

while the third term is a coherence energy that depends on the diabatic coupling $V$ and the coherence $\rho_{12}$ :

$$
E_{c o h}=2 \operatorname{Re} \iint V \rho_{12} d q d p
$$

The sum of the three terms is a constant of the motion. It is important to note that the diagonal energy contributions $E_{1}+E_{2}$ is not conserved. Energy is "stored" in the coherence while the states are interacting and the system is undergoing transitions. 
In order to establish that conservation of total energy is obeyed by the semiclassical Liouville formalism in the diabatic representation, we now show that the time derivative of $E$ vanishes under the semiclassical equations of motion. We have

$$
\frac{d E}{d t}=\iint\left(H_{11} \frac{\partial \rho_{11}}{\partial t}+H_{22} \frac{\partial \rho_{22}}{\partial t}+2 V \frac{\partial \operatorname{Re} \rho_{12}}{\partial t}\right) d q d p .
$$

This consists of three terms:

$$
\begin{aligned}
\frac{d E_{1}}{d t} & =\iint H_{11} \frac{\partial \rho_{11}}{\partial t} d q d p \\
\frac{d E_{2}}{d t} & =\iint H_{22} \frac{\partial \rho_{22}}{\partial t} d q d p \\
\frac{d E_{c o h}}{d t} & =2 \iint V \frac{\partial \operatorname{Re} \rho_{12}}{\partial t} d q d p .
\end{aligned}
$$

The equations of motion are given by

$$
\begin{gathered}
\frac{\partial \rho_{11}}{\partial t}=\left\{H_{11}, \rho_{11}\right\}+\left\{V, \operatorname{Re} \rho_{12}\right\}-\frac{2 V}{\hbar} \operatorname{Im} \rho_{12} \\
\frac{\partial \rho_{22}}{\partial t}=\left\{H_{22}, \rho_{22}\right\}+\left\{V, \operatorname{Re} \rho_{12}\right\}+\frac{2 V}{\hbar} \operatorname{Im} \rho_{12} \\
\frac{\partial \rho_{12}}{\partial t}=\left(\hat{\mathcal{L}}_{0}-i \omega\right) \rho_{12}+\frac{1}{2}\left\{V, \rho_{11}+\rho_{22}\right\}+\frac{i}{\hbar} V\left(\rho_{11}-\rho_{22}\right)
\end{gathered}
$$

or taking real and imaginary parts,

$$
\begin{aligned}
& \frac{\partial \operatorname{Re} \rho_{12}}{\partial t}=\left\{H_{0}, \operatorname{Re} \rho_{12}\right\}+\omega \operatorname{Im} \rho_{12}+\frac{1}{2}\left\{V, \rho_{11}+\rho_{22}\right\} \\
& \frac{\partial \operatorname{Im} \rho_{12}}{\partial t}=\left\{H_{0}, \operatorname{Im} \rho_{12}\right\}-\omega \operatorname{Re} \rho_{12}+\frac{1}{\hbar} V\left(\rho_{11}-\rho_{22}\right) .
\end{aligned}
$$

We can evaluate the time derivatives of the energy contributions using the equations of motion, giving:

$$
\frac{d E_{1}}{d t}=\iint H_{11}\left(\left\{H_{11}, \rho_{11}\right\}+\left\{V, \operatorname{Re} \rho_{12}\right\}-\frac{2 V}{\hbar} \operatorname{Im} \rho_{12}\right) d q d p
$$




$$
\begin{gathered}
\frac{d E_{2}}{d t}=\iint H_{22}\left(\left\{H_{22}, \rho_{22}\right\}+\left\{V, \operatorname{Re} \rho_{12}\right\}+\frac{2 V}{\hbar} \operatorname{Im} \rho_{12}\right) d q d p \\
\frac{d E_{c o h}}{d t}=2 \iint V\left(\left\{H_{0}, \operatorname{Re} \rho_{12}\right\}+\omega \operatorname{Im} \rho_{12}+\frac{1}{2}\left\{V, \rho_{11}+\rho_{22}\right\}\right) d q d p .
\end{gathered}
$$

First we note that

$$
\begin{array}{r}
\iint H_{11}\left\{H_{11}, \rho_{11}\right\} d q d p=-\iint \rho_{11}\left\{H_{11}, H_{11}\right\} d q d p=0 \\
\iint H_{22}\left\{H_{22}, \rho_{22}\right\} d q d p=-\iint \rho_{22}\left\{H_{22}, H_{22}\right\} d q d p=0 \\
\iint V\left\{V, \rho_{11}+\rho_{22}\right\} d q d p=-\iint\left(\rho_{11}+\rho_{22}\right)\{V, V\} d q d p=0,
\end{array}
$$

where we have integrated by parts and used the vanishing of $\rho_{11}$ and $\rho_{22}$ at the phase space boundary. We thus have

$$
\frac{d}{d t}\left(E_{1}+E_{2}\right)=2 \iint\left(H_{0}\left\{V, \operatorname{Re} \rho_{12}\right\}-\omega V \operatorname{Im} \rho_{12}\right) d q d p
$$

where we have used $H_{11}+H_{22}=2 H_{0}$ and $H_{11}-H_{22}=\hbar \omega$, and

$$
\frac{d E_{c o h}}{d t}=2 \iint\left(V\left\{H_{0}, \operatorname{Re} \rho_{12}\right\}+\omega V \operatorname{Im} \rho_{12}\right) d q d p .
$$

The terms $d\left(E_{1}+E_{2}\right) / d t$ and $d E_{c o h} / d t$ are separately nonzero. This illustrates the important fact that the diagonal energy $E_{1}+E_{2}$ is not a conserved quantity in electronic transitions. The total energy however, is rigorously conserved. Noting that

$$
\iint V\left\{H_{0}, \operatorname{Re} \rho_{12}\right\} d q d p=-\iint H_{0}\left\{V, \operatorname{Re} \rho_{12}\right\} d q d p
$$

we see that the total time derivative of $E$ vanishes:

$$
\frac{d E}{d t}=\frac{d}{d t}\left(E_{1}+E_{2}\right)+\frac{d E_{c o h}}{d t}=0
$$

and so the total energy $E=\operatorname{Tr} H \rho$ is a constant of the semiclassical equations of motion. 
We can repeat the analysis in the adiabatic representation. The Hamiltonian and density matrix in the semiclassical limit are

$$
H=\left(\begin{array}{cc}
H_{++} & -i \hbar d \frac{p}{m} \\
i \hbar d \frac{p}{m} & H_{--}
\end{array}\right)
$$

and

$$
\rho=\left(\begin{array}{cc}
\rho_{++} & \rho_{+-} \\
\rho_{-+} & \rho_{--}
\end{array}\right),
$$

respectively. As before, the total energy of the system is given by the trace of the product of $H$ and $\rho$, now in the adiabatic representation:

$$
E=\operatorname{Tr} H \rho=\iint\left(H_{++} \rho_{++}+H_{--} \rho_{--}-2 \hbar d \frac{p}{m} \operatorname{Im} \rho_{+-}\right) d q d p .
$$

The energy is a sum of three terms: $E=E_{+}+E_{-}+E_{c o h}$. The first terms are the average energies of the system on state $|+\rangle$ and state $|-\rangle$, respectively:

$$
\begin{aligned}
& E_{+}=\iint H_{++} \rho_{++} d q d p \\
& E_{-}=\iint H_{--} \rho_{--} d q d p
\end{aligned}
$$

while the third term is a coherence energy that depends on the adiabatic coupling $-i \hbar d \frac{p}{m}$ and the coherence $\rho_{+-}$.

$$
E_{c o h}=-2 \hbar \operatorname{Im} \iint d \frac{p}{m} \rho_{+-} d q d p .
$$

The time derivative of the total adiabatic energy is

$$
\frac{d E}{d t}=\iint\left(H_{++} \frac{\partial \rho_{++}}{\partial t}+H_{--} \frac{\partial \rho_{--}}{\partial t}-2 \hbar d \frac{p}{m} \frac{\partial \operatorname{Im} \rho_{+-}}{\partial t}\right) d q d p .
$$

This consists of three terms:

$$
\begin{aligned}
\frac{d E_{+}}{d t} & =\iint H_{++} \frac{\partial \rho_{++}}{\partial t} d q d p \\
\frac{d E_{-}}{d t} & =\iint H_{--} \frac{\partial \rho_{--}}{\partial t} d q d p
\end{aligned}
$$




$$
\frac{d E_{c o h}}{d t}=-2 \hbar \iint d \frac{p}{m} \frac{\partial \operatorname{Im} \rho_{+-}}{\partial t} d q d p .
$$

The equations of motion are given by

$$
\begin{gathered}
\frac{\partial \rho_{++}}{\partial t}=\left\{H_{++}, \rho_{++}\right\}-2 d \frac{p}{m} \operatorname{Re} \rho_{+-}-\hbar\left\{d \frac{p}{m}, \operatorname{Im} \rho_{+-}\right\} \\
\frac{\partial \rho_{--}}{\partial t}=\left\{H_{--}, \rho_{--}\right\}+2 \frac{p}{m} \operatorname{Re} \rho_{+-}-\hbar\left\{d \frac{p}{m}, \operatorname{Im} \rho_{+-}\right\} \\
\frac{\partial \rho_{+-}}{\partial t}=\left\{H_{o}, \rho_{+-}\right\}-i \omega \rho_{+-}+d \frac{p}{m}\left(\rho_{++}-\rho_{--}\right)-\frac{i \hbar}{2}\left\{d \frac{p}{m}, \rho_{++}+\rho_{--}\right\}
\end{gathered}
$$

or taking real and imaginary parts,

$$
\begin{gathered}
\frac{\partial \operatorname{Re} \rho_{+-}}{\partial t}=\left\{H_{o}, \operatorname{Re} \rho_{+-}\right\}+\omega \operatorname{Im} \rho_{+-}+d \frac{p}{m}\left(\rho_{++}-\rho_{--}\right) \\
\frac{\partial \operatorname{Im} \rho_{+-}}{\partial t}=\left\{H_{o}, \operatorname{Im} \rho_{+-}\right\}-\omega \operatorname{Re} \rho_{+-}-\frac{\hbar}{2}\left\{d \frac{p}{m}, \rho_{++}+\rho_{--}\right\} .
\end{gathered}
$$

We can evaluate the time derivatives of the energy contributions using the equations of motion, giving:

$$
\begin{gathered}
\frac{d E_{+}}{d t}=\iint H_{++}\left(\left\{H_{++}, \rho_{++}\right\}-2 d \frac{p}{m} \operatorname{Re} \rho_{+-}-\hbar\left\{d \frac{p}{m}, \operatorname{Im} \rho_{+-}\right\}\right) d q d p \\
\frac{d E_{-}}{d t}=\iint H_{--}\left(\left\{H_{--}, \rho_{--}\right\}+2 \frac{p}{m} \operatorname{Re} \rho_{+-}-\hbar\left\{d \frac{p}{m}, \operatorname{Im} \rho_{+-}\right\}\right) d q d p \\
\frac{d E_{c o h}}{d t}=-2 \hbar \iint d \frac{p}{m}\left(\left\{H_{o}, \operatorname{Im} \rho_{+-}\right\}-\omega \operatorname{Re} \rho_{+-}-\frac{\hbar}{2}\left\{d \frac{p}{m}, \rho_{++}+\rho_{--}\right\}\right) d q d p .
\end{gathered}
$$

As in the diabatic representation, simplifications occur due to vanishing terms, giving

$$
\frac{d}{d t}\left(E_{+}+E_{-}\right)=-2 \hbar \iint\left(\omega d \frac{p}{m} \operatorname{Re} \rho_{+-}+H_{0}\left\{d \frac{p}{m}, \operatorname{Im} \rho_{+-}\right\}\right) d q d p
$$

where we have used $H_{++}+H_{--}=2 H_{0}$ and $H_{++}-H_{--}=\hbar \omega$, and

$$
\frac{d E_{c o h}}{d t}=2 \hbar \iint\left(\omega d \frac{p}{m} \operatorname{Re} \rho_{+-}-d \frac{p}{m}\left\{H_{0}, \operatorname{Im} \rho_{+-}\right\}\right) d q d p .
$$


Again, the sum of the diagonal energies is not a constant of the motion. Using

$$
\iint d \frac{p}{m}\left\{H_{0}, \operatorname{Im} \rho_{12}\right\} d q d p=-\iint H_{0}\left\{d \frac{p}{m}, \operatorname{Im} \rho_{12}\right\} d q d p
$$

we see that the total time derivative of $E$ vanishes:

$$
\frac{d E}{d t}=\frac{d}{d t}\left(E_{+}+E_{-}\right)+\frac{d E_{c o h}}{d t}=0
$$

and so the total energy $E=\operatorname{Tr} H \rho$ is a constant of the semiclassical equations of motion in the adiabatic representation.

\section{Discussion}

The analysis of the semiclassical limit equations of motion for the density matrix describing nonadiabatic electronic transitions presented above highlights a number of key features. We close by discussing these features from the perspective of trajectory-based methods for simulating molecular dynamics with electronic transitions.

Locality of the equations of motion in phase space.

The semiclassical Liouville equations, Eq. (15)-(17) for the diabatic representation and Eq. (36)-(38) for the adiabatic representation, are local in phase space. In either representation, the change in time of $\rho_{i j}(q, p, t)$ at the point $(q, p)$ depends on the set of functions $\rho_{i^{\prime}, j^{\prime}}$ and their derivatives at that point. For the populations, the terms in the equations of motion that do not have vanishing phase space trace and therefore induce population transfer between the electronic states exactly mirror each other point-by-point in phase space. Consider an element of population transfer from state 1 to state 2 in the diabatic representation. According to Eq. (15), the decrease of $\rho_{11}(q, p, t)$ at the point $(q, p)$ and time $t$ during the interval $\Delta t$ is given by $\Delta \rho_{11}=-2 V \rho_{12}(q, p, t) \Delta t / \hbar$. The increase of $\rho_{22}(q, p, t)$ at that point in phase space during $\Delta t$ is given by Eq. (16) as $\Delta \rho_{22}=+2 V \rho_{12}(q, p, t) \Delta t / \hbar$, which exactly mirrors the transformation-inducing change of $\rho_{11}$. (Additional changes in the diagonal density matrix elements are caused by the $\left\{V, \operatorname{Re} \rho_{12}\right\}$ term; this affects both populations equally and does not break the locality of population transfer).

What does this suggest for a trajectory-based descrption of electronic transitions? The popular FSSH method represents the evolving phase space 
distribution functions by an ensemble of trajectories, and population transfer is modeled by stochastic hops of the trajectories between the states. The point-by-point locality of the underlying equation for the states represented by that ensemble suggests that these hops should be made locally in phase space as well. The FSSH algorithm as typically implemented does not do this. Rather, it rescales the momenta of hopping trajectories to impose strict energy conservation at the individual trajectory level. The result is a nonlocal dynamics of population transfer, where probability leaving one surface at point $(q, p)$ appears on the other surface at a different point $(q, p+\delta p)$, where $\delta p$ is a momentum shift made to impose energy conservation.

Momentum nonlocality in the transformation between electronic representations.

Nonlocality does appear in the semiclassical theory, not as part of the dynamics of electronic transition but rather in the transformation equations relating the diabatic and adiabatic representations of the electronic states. This arises due to the coordinate dependence of the unitary transformation between the electronic bases. When the Wigner transform of the density matrix elements in the position representation is performed, this dependence causes a heterodyne-like phenomenon that relates Wigner distributions in different representations nonlocally in phase space. In particular, shifts of the momentum variable emerge which depend on both Planck's constant and the nonadiabatic coupling matrix element $d(q)$.

The energy budget of coherent nonadiabatic dynamics.

The total energy of the evolving coupled system in the semiclassical limit is given by $E=\operatorname{Tr} H \rho$, where the trace is the sum of the phase space integrals of the diagonal elements of the matrix $H \rho$. This is true in both the diabatic and adiabatic representations. By employing the semiclassical equations of motion, we showed above that the total energy is a sum of the diagonal energies plus a term that we indicate $E_{c o h}$, which is a contribution that is nonzero whenever electronic coherence and coupling are present. This total energy is a constant of the motion under the semiclassical evolution. Again, this is true for both diabatic and adiabatic representations.

It is important to note that the sum of the diagonal energies in both cases is not conserved. During electronic transitions, energy is "stored" in the coherence between the states. This observation has important implications for modeling electronic transitions with trajectories. In particular, the analysis 
suggests that, when designing trajectory-based methods for simulating the molecular dynamics with electronic transitions, strict energy conservation of the trajectories should not be imposed during hops between the states. Even at the ensemble level, the total diagonal energies do not sum to the conserved total energy, due to the contribution of $E_{c o h}$.

The phase space locality of the equations of motion and the absence of a strict trajectory level requirement for energy conservation suggest that surface hopping methods without momentum rescaling have a firm theoretical foundation. The majority of previous work on the surface hopping approach have assumed the necessity of strict energy conservation at the trajectory level $[7,8,9,10,11,12,13]$, with the work of Ando and Santer being a notable exception [30].

In the limit of rapid decoherence, trajectory independence and energy conservation are sensible and efficient approximations to make. Indeed, hopping of independent trajectories with momentum rescaling as commonly practiced has demonstrated its accuracy and utility in a range of physical systems. For manifestly quantum coherent processes, however, a more rigorous treatment becomes important. The use of trajectories to model manifestly nonclassical quantum effects will, of necessity, involve generalizing strict classical features of the dynamics. We have discussed this previously in the context of the so-called "entangled trajectory" method for modeling quantum tunneling $[35,26,36]$ or diffusive motion $[37,38]$. Although different in detail, nonadiabatic electronic transitions share with quantum tunneling an intrinsically nonclassical element. In future publications, we will describe a trajectorybased approach to nonadiabatic dynamics that implements the formal ideas outlined in this paper in a quantitative method that is both accurate and efficient [39].

\section{Acknowledgments}

We are grateful to Shaul Mukamel, Vladimir Mandelshtam, and Greg Ezra for helpful discussions. Stimulating discussions at the UCI Liquid Theory Lunch (LTL) and the Telluride Science Research Center (TSRC) are also acknowledged. This work is an outgrowth of research supported by the National Science Foundation under CHE-0614005 and CHE-0802913. 


\section{References}

[1] M. H. Beck, A. Jackle, G. A. Worth, H. D. Meyer, The multiconfiguration time-dependent hartree (mctdh) method: A highly efficient algorithm for propagating wavepackets, Phys. Rep. 324 (2000) 1.

[2] H.-D. Meyer, Studying molecular quantum dynamics with the multiconfiguration time-dependent hartree method, Wiley Interdisciplinary Reviews: Computational Molecular Science 2 (2012) 351-374.

[3] M. P. Allen, D. J. Tildesley, Computer Simulation of Liquids, Clarendon Press, Oxford, 1987.

[4] G. Ciccotti, D. Frenkel, I. R. McDonald (Eds.), Simulation of Liquids and Solids, North Holland, New York, 1987.

[5] H. Goldstein, Classical Mechanics, 2nd Edition, Addison-Wesley, Reading, 1980.

[6] Z. Li, R. Zadoyan, V. A. Apkarian, C. C. Martens, Femtosecond many-body dynamics of caging. experiment and simulation of $\mathrm{I}_{2}$ photodissociation-recombination in solid Ar, J. Phys. Chem. 99 (1995) 7453-7465.

[7] J. C. Tully, Molecular dynamics with electronic transitions, J. Chem. Phys. 93 (1990) 1061.

[8] J. C. Tully, Perspective: Nonadiabatic dynamics theory, J. Chem. Phys. 137 (2012) 22A301.

[9] J. E. Subotnik, N. Shenvi, A new approach to decoherence and momentum rescaling in the surface hopping algorithm, J. Chem. Phys. 134 (2011) 024105.

[10] H. M. Jaeger, S. Fischer, O. V. Prezhdo, Decoherence-induced surface hopping, J. Chem. Phys. 137 (2012) 22A545.

[11] J. E. Subotnik, W. Ouyang, B. R. Landry, Can we derive Tully's surfacehopping algorithm from the semiclassical quantum Liouville equation? Almost, but only with decoherence, J. Chem. Phys. 139 (21) (2013) 214107. 
[12] B. F. E. Curchod, I. Tavernelli, On trajectory-based nonadiabatic dynamics: Bohmian dynamics versus trajectory surface hopping, J. Chem. Phys. 138 (2013) 184112.

[13] H.-T. Chen, D. R. Reichman, On the accuracy of surface hopping dynamics in condensed phase non-adiabatic problems, The Journal of Chemical Physics 144 (9) (2016) 094104-10.

[14] T. J. Martinez, R. D. Levine, First-principles molecular dynamics on multiple electronic states: A case study of nai, J. Chem. Phys. 105 (1996) 6334.

[15] M. D. Hack, A. M. Wensmann, D. G. Truhlar, T. J. Martinez, Comparison of full multiple spawning, trajectory surface hopping, and converged quantum mechanics for electronically nonadiabatic dynamics, J. Chem. Phys. 115 (2001) 1172.

[16] X. Sun, W. H. Miller, Semiclassical initial value representation for electronically nonadiabatic molecular dynamics, J. Chem. Phys. 106 (1997) 6346 .

[17] W. H. Miller, The semiclassical initial value representation: A potentially practical way for adding quantum effects to classical molecular dynamics simulations, J. Phys. Chem. A 105 (2001) 2942.

[18] C. L. Lopreore, R. E. Wyatt, Electronic transitions with quantum trajectories. ii, J. Chem. Phys. 116 (2002) 1228-1238.

[19] S. Bonella, D. F. Coker, A semiclassical limit for the mapping hamiltonian approach to electronically nonadiabatic dynamics, J. Chem. Phys. 114 (2001) 7778.

[20] S. Bonella, D. Montemayor, D. F. Coker, Linearized path integral approach for calculating nonadiabatic time correlation functions., Proc. Natl. Acad. Sci. 102 (2005) 6715-6719.

[21] S. Bonella, D. F. Coker, LAND-map, a linearized approach to nonadiabatic dynamics using the mapping formalism., J. Chem. Phys. 122 (2005) 194102. 
[22] C. C. Martens, J. Y. Fang, Semiclassical-limit molecular dynamics on multiple electronic surfaces, J. Chem. Phys. 106 (1997) 4918-4930.

[23] A. Donoso, C. C. Martens, Simulation of coherent nonadiabatic dynamics using classical trajectories, J. Phys. Chem. A 102 (1998) 4291-4300.

[24] A. Donoso, C. C. Martens, Semiclassical multi-state liouville dynamics in the adiabatic representation, J. Chem. Phys. 112 (2000) 3980-3989.

[25] A. Donoso, D. Kohen, C. C. Martens, Simulation of nonadiabatic wavepacket interferometry using classical trajectories, J. Chem. Phys. 112 (2000) 7345-7354.

[26] A. Donoso, C. C. Martens, Classical trajectory-based approaches to solving the quantum liouville equation, Int. J. Quantum Chem. 87 (2002) $1348-1360$.

[27] R. Kapral, G. Ciccotti, Mixed quantum-classical dynamics, J. Chem. Phys. 110 (1999) 8919.

[28] G. Hanna, R. Kapral, Nonadiabatic dynamics of condensed phase rate processes., Acc. Chem. Res. 39 (2006) 21-27.

[29] K. Ando, Non-adiabatic couplings in liouville description of mixed quantum-classical dynamics, Chem. Phys. Lett 360 (2002) 240-242.

[30] K. Ando, M. Santer, Mixed quantum-classical liouville molecular dynamics without momentum jump, J. Chem. Phys. 118 (2003) 1039910406.

[31] D. A. McQuarrie, Statistical Mechanics, HarperCollins, New York, 1976.

[32] M. Hillery, R. F. O'Connell, M. O. Scully, E. P. Wigner, Distributionfunctions in physics-fundamentals, Phys. Rep. 106 (1984) 12.

[33] W. P. Schleich, Quantum Optics in Phase Space, Wiley-VCH, Berlin, 2001.

[34] I. G. Ryabinkin, C.-Y. Hsieh, R. Kapral, A. F. Izmaylov, Analysis of geometric phase effects in the quantum-classical Liouville formalism, J. Chem. Phys. 140 (8) (2014) 084104. 
[35] A. Donoso, C. C. Martens, Quantum tunneling using entangled classical trajectories, Phys. Rev. Lett. 87 (2001) 223202.

[36] A. Donoso, Y. Zheng, C. C. Martens, Simulation of quantum processes using entangled trajectory molecular dynamics, J. Chem. Phys. 119 (2003) 5010.

[37] A. Donoso, C. C. Martens, Solution of phase space diffusion equations using interacting trajectory ensembles, J. Chem. Phys. 116 (2002) 10598.

[38] P. Hogan, A. V. Wart, A. Donoso, C. C. Martens, Solving evolution equations using interacting trajectory ensembles, Chem. Phys. 370 (2010) 20.

[39] C. C. Martens, Surface Hopping by Consensus, J. Phys. Chem. Lett. (submitted). 